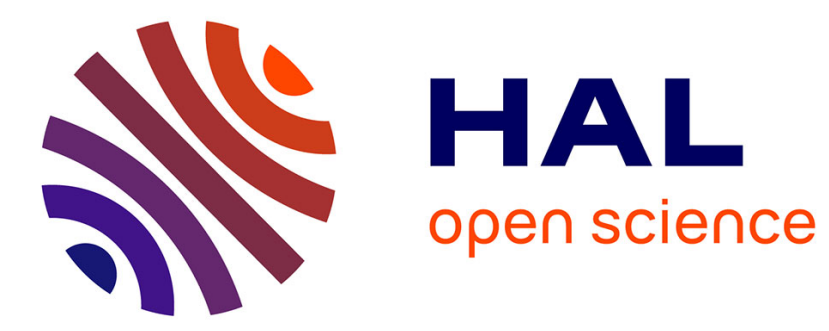

\title{
Deux chenets de pierre à tête de bélier découverts à Riec-sur-Belon (Finistère)
}

\author{
Patrick Galliou, Michel Clément
}

\section{To cite this version:}

Patrick Galliou, Michel Clément. Deux chenets de pierre à tête de bélier découverts à Riec-sur-Belon (Finistère). Gallia - Fouilles et monuments archéologiques en France métropolitaine, 1981, 39 (2), pp.235-238. 10.3406/galia.1981.1833 . hal-01940650

\section{HAL Id: hal-01940650 \\ https://hal.science/hal-01940650}

Submitted on 27 Feb 2020

HAL is a multi-disciplinary open access archive for the deposit and dissemination of scientific research documents, whether they are published or not. The documents may come from teaching and research institutions in France or abroad, or from public or private research centers.
L'archive ouverte pluridisciplinaire HAL, est destinée au dépôt et à la diffusion de documents scientifiques de niveau recherche, publiés ou non, émanant des établissements d'enseignement et de recherche français ou étrangers, des laboratoires publics ou privés.

\section{(ㅇ)(1) $\$$}

Distributed under a Creative Commons Attribution - NonCommercial - NoDerivatives| 4.0 


\title{
DEUX CHENETS DE PIERRE À TÊTE DE BÉLIER DÉCOUVERTS À RIEC-SUR-BELON (Finistère)
}

\author{
par Patrick GALLIOU et Michel CLÉMENT
}

Ces deux objets ${ }^{1}$ furent exhumés au lieu-dit 'l'alhiern (commune de Riec-sur-Belon, Finistère) à quelques années d'intervalle (1970 et 1979), dans une parcelle (section ZK, no 14) où les labours ramènent en surface tuiles à rebords et céramiques (anse d'amphore vinaire italique Dressel I, lerra nigra, etc.). Ils furent confiés à la Direclion des Antiquités historiques de Bretagne, qui nous chargea de leur étude. La trouvaille conjointe d'une meule rotative et d'abondantes scories de fer donne à penser qu'existait en ce lieu un établissement important, dont on peut situer la naissance au $1^{\text {er }}$ siècle avant notre ère ${ }^{2}$.

Les deux chenets - malheureusement brisés au niveau du cou et quelque peu endommagés par les charrues -... ont été taillés dans une roche locale, le leucogranite sud-armoricain, dont les gros grains de quartzite rendent le travail malaisé. Bien que légèrement différents dans le détail, ces deux objets semblent sortir du même atelier : ils ont approximativement la même taille (long. de la tête : $27 \mathrm{~cm}$ ) et leur modelé naturaliste les distingue des autres représentations du même type, en pierre ou en terre cuite, à l'aspect souvent stylisé ${ }^{3}$. Le rendu anatomique est relativement satisfaisant, bien que l'artisan ait renoncé à figurer les

l La découverte a été signalée par R. SAvquer, Chronique d'archéologie antique et médiévale, dans Bull. de la Soc. archéologique du Finistère, CVI, 1978, p. $79-80$.

2 I.e toponyme breton "Talhiarn" signifie : "le bout de la forge". boucles de la toison; les cornes, sous lesquelles s'attachent des bourrelets symétriques descendant le long du cou, s'enroulent en spirales finement dessinées et le tracé des yeux . légèrement saillants - répond aux canons de l'esthétique gauloise (fig. 1 et 2 ).

Une étude récente n'a guère recensé qu'une dizaine d'objets similaires, répartis sur le territoire de la Celtique, de la Belgique et de l'Aquitaine, à La Graufesenque (trois exemplaires en grès), Saint-Bertrand-de-Comminges (calcaire), Les Fins-d'Annecy (deux exemplaires en grès), Bavay (pierre blanche), Zürich (pierre commune), IIontreuil-Bellay (grès) et Vannes (granite) $)^{5}$, auxquels vient s'ajouter un chenet de grès recueilli à Ehl (Bas-Rhin) ${ }^{6}$. On ne peut cependant douter que cette brève série relève d'un modèle fort bien défini par les travaux que lui ont consacrés plusieurs archéologues et historiens de l'art, celui des chenets occidentaux à têle de bélier?. La grande majorité des

3 Il suffit de comparer au dessin des chenets de Riec ceux des chenets publiés par J. Déchelette, Le bélier consacré aux divinités domestiques sur les chenets gaulois, dans Revue archéologique, XXXIII, 1898 , p. $63-81$ et p. 245-262.

4 Cf. par exemple: G. Gaudrox, Trois letes de bélier inédites, sommels de chenets d'argile de tradition gauloise, dans Bull. de la Soc. nationale des Antiquaires de France, 1957, p. 137.

5 Cf. R. Gavelle, Sur un chenet à tète de bélier en calcaire trouvé $\dot{a}$ Lugdunum Convenarum, dans Ogam, XII, 1960, p. 296-302; ibid., Chenels à tèle de bélier en pierre, ibid., p. 428-430.

6 C.f. Gallia, XXVIII, 1970, p. 342-343.

7 C. en particulier : J. DÉcheretere, op. cil.; 
objets de ce groupe étant faits de terre cuite, il n'est d'ailleurs pas impossible que les exemplaires taillés dans la pierre constituent des objets de luxe $e^{8}$, à moins qu'il ne s'agisse d'une imitation - - selon les traditions locales -. de modèles importés.

Les chenets de pierre et de céramique mis au jour dans l'Quest de l'Europe comportent tous une lourde base à section triangulaire ou quadrangulaire, à l'extrémité de laquelle se dresse une gaine verticale. De cette dernière émerge un protomé de bélier (ou de cheval), souvent très stylisé ${ }^{9}$. Des bourrelets latéraux caractérisent aussi la plupart de ces tètes animales : on peut y reconnaître "les guirlandes dont on parait les victimes et les images des divinités domestiques $\|^{10}$, les forts rouleaux de laine en bourre suspendus aux cornes des animaux promis au sacrifice.

Les chenets de Riec-sur-Belon, s'ils se distinguent par leur aspect réaliste. se singularisent aussi par leur taille. La majorité des chenets gaulois ne dépassent quère, en effet, $25 \mathrm{~cm}$ de hauteur, la tête de l'animal étant de la grosseur du poing. Or, si l'on admet que les proportions des chenets de Riec étaient semblables à celles des modèles de céramique, on doit supposer que ceux-ci avaient environ 7.5 cm de haut, ce qui les classe, avec l'exemplaire de Zürich mentionné ci-dessus, parmi les plus grands d'Occident"1'.

J. Décheletre, Manuel d'archéologie préhislorique, cellique el gallo-romaine. II. Archéologie celtique ou protohistorique. Troisième partic. Second Age du Fer, ou époque de La Tène, Paris, 1914, p. 1399-1428; G. Faider-Feytmans, Le culle cellique du foyer dans la cilé des Nerviens, dans Documents el rapports de la Sociélé royale paléonlologique et archéologique de Charleroi, 47, 1950, p. 99-117; S. DE LAET, Données nouvelles sur la survivance du culle cellique du foyer dans la cité des Nerviens à l'époque romaine, dans Estudios dedicados al Professor Dr. Luis Pericol, 1973, p. 323-331.

8 C'est lopinion exprimé a propos de cerlains chenets découverts en Bulgarie; ef. T. Gremasimov, Chenets celliques en Bulgarie, dans Latomus, XXXI, 1972 , p. 722 .

9 Cif par exemple le chenet de granite mis au jour $\dot{a}$ Vannes : L. MArsitce, Chenet gallo-romain à lète de bélier, en gramite, dans Bull. de la Soc. polymathique du Morbihan, 1927, p. 105-107.

10 J. 1) в́снелетте, Le bélier..., op. cit., p. 257.

11 Certains chenels de Bulgarie dépassent toutefois le metre.
Il n'est d'ailleurs pas impossible qu'à ces différences de taille correspondent des fonctions lechniques différentes. Les chenets étaient sans doute toujours utilisés par paires, et placés perpendiculairement au mur contre lequel s'adossait le foyer, mais on peut admettre que les chenets bas n'ont servi qu'à "limiter le foyer, à en retenir les cendres et surtout ¿ faciliter la combustion des bûches "12, alors que "les landiers à gaine élevée faisaient office de hastiers $\|^{13}$, et que les petites cavités ménagées dans les parois latérales permettaient de loger les extrénités des broches à ròtir. Les chenets de Riec, dont l'exemplaire découvert en 1979 porte les traces d'un feu violent, ont done pu servir à ce dernier usage.

Selon l'hypothèse formulée au siècle dernier par J. Déchelette ${ }^{14}$ et aujourd'hui communément admise ${ }^{15}$, ces objets, étroitement associés au foyer domestique et portant des figurations a connotation religieuse, ne peuvent aroir eu un ròle purement utilitaire et fonctionnel. Le bélier, dont la mythologie souligne la force, le courage et la fécondité, apparait donc sur les chenets comme "le symbole du sacrifice offert aux àmes des ancêtres sur l'autel du foyer $»^{16}$ : il est, en effet, exact qu'un certain nombre de ces objets, découverts in silu, proviennent d'habitations privées dont ils ornaient probablement le foyer ${ }^{17}$. Mais, comme l'a fait remarquer G. Gaudron, les chenets mis au jour en Gaule sont relativement peu nombreux et il n'est absolument pas certain qu'ils aient constitué l'accessoire obligatoire de chaque foyer individuel, ni qu'ils renvoient dans tous les cas a un culte focal purement familial.

Il n'est pas étonnant, d'autre part, que soient déposés dans la tombe des objets culinaires devant permettre au défunt de se nourrir dans l'au-delà et faisant pendant aux ustensiles en usage chez les vivants. Ces pratiques funéraires,

12 J. DÉchli.tте, Le bélier..., op. cil., p. 80.

13 J. DÉchelette, ibid., p. 80.

I.4 J. Dв́nkletTk, Le bélier..., op. cil.

15 Cf. S. DE I.AET, op. cil., p. 323.

16 J. Déchelette, Le bélier..., op. cil., p. 252.

17 A Besançon: Gallia, XI, 1953, p. 136; Quimper: Gallia, 33, 1975, fig. 30, 9; Ehl : Gallia, XXVIII, 19711,1 . $3.12-3.43$. 


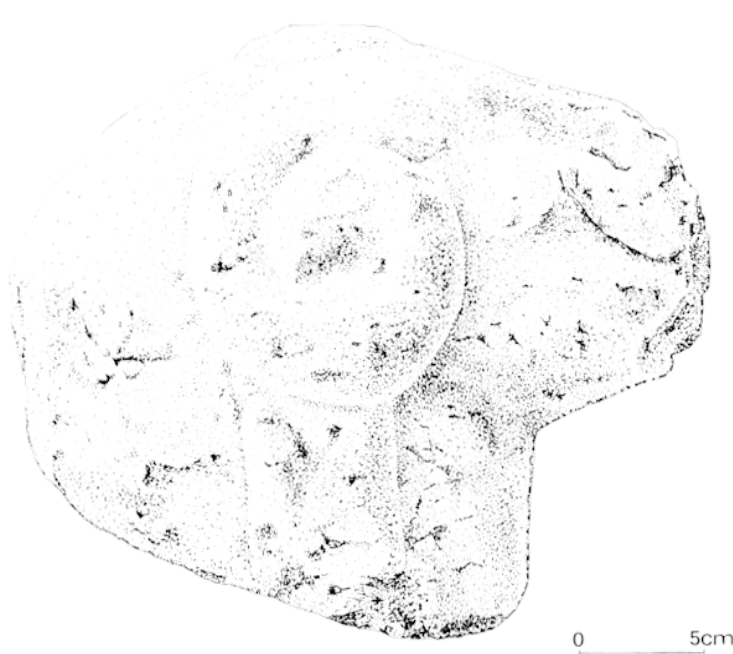

attestees a haute époque dans l'()rient méditerranéen ${ }^{18}$, semblent s'etre lentement répandues dans loute l'Europe occidentale pendant les périodes du Hallstalt el de La T’óne et n'avoir guère survécu, dans la plupart des régions, a la poussée de la civilisalion romaine. Elles se rencontrent cependant, aux derniers lemps de l'Indépendance et lors des deux premiers siecles de nolre ire, dans deux zones habitées par des descendants des Belgae de l'Age du Fer, che\% les tribus belges d'Angleterre d'une part ${ }^{19}$ el rhe\% les tribus de Gaule Belgique d'autre part ${ }^{20}$. les lombes de ces regions

Ix lit. lih. Pusanu, sur quelques connexions des domaines de la prolohistoire de lOrienl à lOocident médilerranien: "slalues-menhirs", chenets de foyers, dans Alli del VI. congress" inlernazionale delle scienze preistorica e proloistorica, Roma, 1962 1965, vol. II,

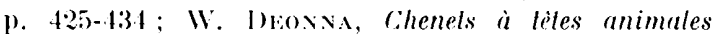
el chenels-navires, dans Reve archóologique de l' lisl, X, 1959, p. 24-37, $\times 1-93,177-191,278-290$.

19 (:t. en parliculier : S. Preciote, Firedogs in Iron Age Britain and beyond, dans J. BoARomax,

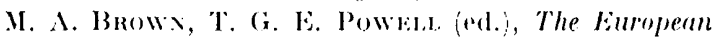
community in laler prehistory. Studies in honour of C. F. C. Ianlies, lomdon, 1971, p. 2.5-270; 1. STran, A La Tine III burial at Weluyn ciarden fily, dans Archacologia, 101, 1967, p. 1-62.

20 (if. en parliculier : G. Famer-Fripuaxs, op. cil.; Chenels gaulois lrouvers dans le Hainaul, dans L'Anliquilé classique, 17, 1948, p. 175-182; Culte celtique du foyer, ibial., 19, 1956, p. $116 ; .1$ propos de chaudrons celtiques, ibid., 21, 1952, p. 409-111; F. Cocntor, le vase culluel de Flavion, ibid., 17, 19.4\%, p. 119-121 ; S. II: I.ANT, op. cil.; Chenel celtique it lile de bilier troulé à laller (Flandre orientale), dalns IIelinium, $\mathrm{N}$, 1975, p. 43-50.

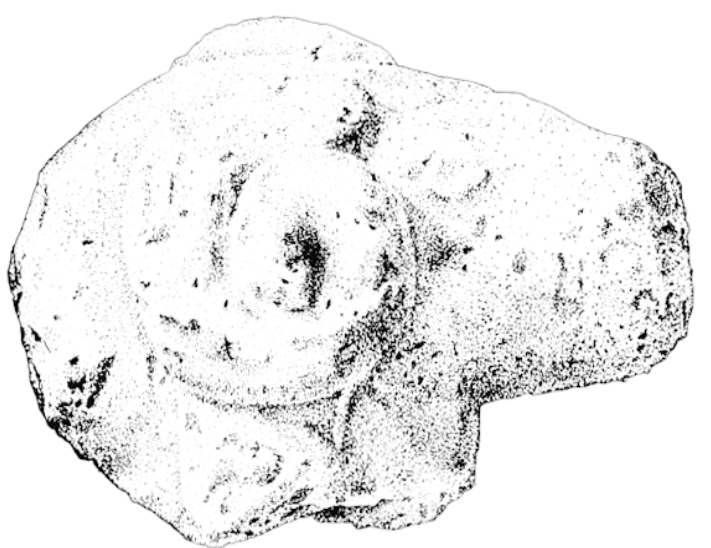

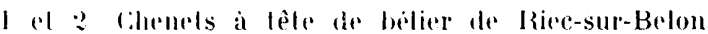
rinisline.

contiennent fréquemment des dépòts funéraires comportant un chaudron de métal, un trépied servant à supporter le chaudron pendant la cuisson, des crémailleres el des chenets de métal ou de céramique. de types d'ailleurs tris diflérents ${ }^{21}$. Dans les tombes belges insulaires on a souvent déposé l'objet lui-mème alors que sur le continent, et che\% les Nerviens en particulier, on l'a remplacé par des modèles miniaturisés, en céramique ${ }^{22}$.

Les sćpultures de ce type sont bien plus rares en fiaule Celtique et l'on ne peul guere comparer aus lombes du mont Beurray qu'une sépulture a incinération. mise au jour au siecle dernier à lostmarch-en-Crozon (Finistere), qui contenail "une lèle de bélier en pierre (sic) rouge brique de grandeur naturelle", placée dans "un vase en terre rouge " enfoui sous une pierre plate à proximité d'une urne cinéraire ${ }^{24}$.

Les chenels occidentaux a tète de bélier apparaissent comme le dernier slade d'une longue évolution dont on peut suive le

$\because 1$ la's "chenets" (firedogs) mis all jour en Anglelere se composent diune structure melallique rectangulaire aux coins de laquelle se dressent des liuges verticales terminés par des têtes animales.

2.2 S. DE LANT, Donnćes nunvelles..., op. cil., p. 327.

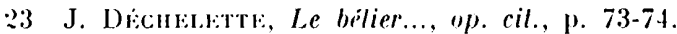

:. A. Janxo, Noles d'archoologie, dans Bull. de la soc. acalémique de Brest, XXIX, 1903-1904, p. 91; 1. J ario, De quelques points interessants de nos côtes: Jinan, Lostmarch et Lesleven en Crozon, daus Bull.

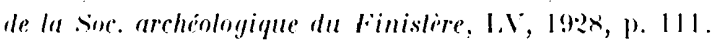


mouvement de l'Italie du nord (civilisations étrusque et villanovienne) au Sud-Est de la Gaule ${ }^{25}$, puis au centre de la Celtique. Lal plupart des objets de ce type découverts en Gaulc scmblent pouvoir être datés des derniers moments de l'Indépendance et du ${ }^{\text {er }}$ siècle de la présence romaine ${ }^{26}$, et leur production ne parait pas avoir survécu à la poussée romanisante de la fin du $\mathrm{i}^{\mathrm{er}}$ siècle ${ }^{27}$. Nous pouvons, selon toute vraisemblance, attribuer les chenets de Riec-sur-Belon à la première moitié de ce siècle.

La présence d'objets de ce type dans l'Ouest de la Gaule n'a rien de surprenant, et il est nécessaire d'étendre (fig. 3) la zone de répartition que leur assignait J. Déchelette ${ }^{28}$ à toute l'Armorique. Les chenets de terre cuite et de pierre sont en effet relativement nombreux à Rezé, port des Pictons, ainsi que sur le territoire des Namnètes a Nantes, Mauves et Blain, mais ils se rencontrent aussi chez les Vénètes à Vannes, les Riedones à Rennes et dans la partie sud de la vaste civilas des Osismes, à Crozon, Quimper et Riec ${ }^{29}$. Il est peu probable que celte répartition essentielle-

25) Italie du nord : ef. J. DÉcueı.eTte, Le bélier..., op. cit., p. 245-248; Sud de la Ganle : Gallia, XII, 1954 , p. 422 ; ibid., XVII, 1959, p. $237-241$; ibill., XXIX, 1971, p. 394 .

26 Besançon : époque julio-claudienne (Gallia, NI, 1953, p. 136); Chateaumeillant époque claudienne (Gallia, XIV, 1961, p. 329-330 ; -.. Quimper : milieu du ier $\mathrm{s}$.

27 Le chenet découvert à Ehl ist cependant daté du début du I $^{\mathrm{e}} \mathrm{s}$. : cf. Gallia, XXVIII, 1970, p. 342-343.

28 J. Déchfiftre, Le bélier..., op. cil., p. 252.

29 Nantes : Lisle dF Dreneuc, Calalogue du Musie archelogique de Nantes, Nantes, 1903, nos 45.1457 ;-- Reze : ibid., nos 79, 79 bis, 143-144; - Mauves : L. Maitra:, Géographie historique et descriptive de la Loire-Inférieure, 1. I : Les villes disparues des Namnites, Nantes, 1893, p. 30-51; -.. Blain : l'objet qui y ful découvert, sorte de gaine verlicale en ceramique, portant d'une part lat représentation d'un dieu cornu debout sur un animal et tenant une bourse de la main droite et d'autre part l'image d'un serpent, a ite considéré comme un élément de chenet : cf. J. Brrs:t.İ́re, Noles archóologiques sur Blain, dans Bull. de la Soc. archénlogique de Nantes et de la Loire-Inférieure, 44, 1903, p. 182 ; Rennes: P. Meriat, Notices d'archeiologie. $\quad \therefore$ Découvertes à Rennes (Ille-el-I'ilaine), dans Annales de Brelagne, L.XIV, 1957, p. 10:; Crozon : A. Jarso, op. cit.; Quimper : R. SANoler, op. cit.

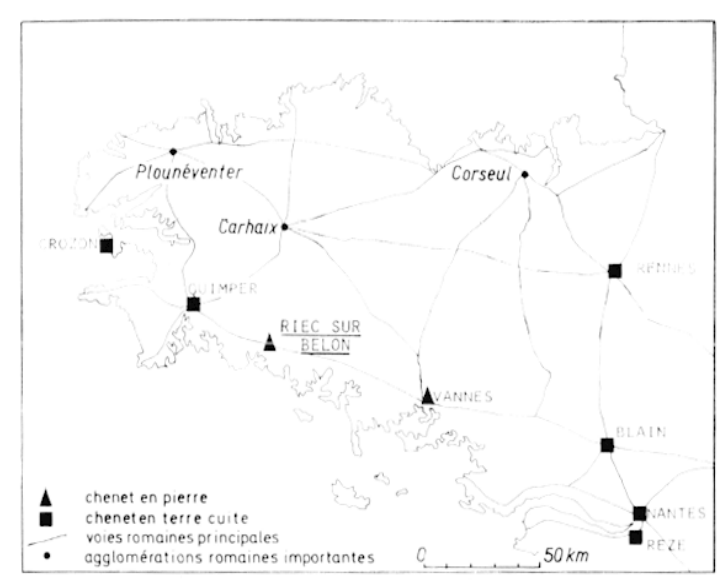

3 Carle do répartition des chenets à tête de bélier découverts en Armorique.

ment méridionale traduise une quelconque unité religieuse et nous préférons y voir l'influence d'échange. commerciaux dont nous commençons à percevoir l'ampleur. Il semble, en effet, que beaucoup de ces chenets de terre cuile aient été produits dans le centre de la Gaule. le long de la Loire et de ses affluents, là où s'installèrent les grands ateliers de potiers des Arvernes ${ }^{30}$, et qu'ils aient été exportés par la Loire vers l'Ouest de la Gaule, tout comme les premières productions céramiques de ces régions, urnes globulaires à bord mouluré d'époque augustéenne ${ }^{31}$, poteries peintes du Forez, sigillée, terra nigra, etc. La carte de répartition de ces céramiques précoces en Armorique coïncide d'ailleurs presque parfaitement avec celle des chenets en terre cuite, ce qui souligne une fois encore l'étroitesse des liens qui unissaient deux des régions les plus "celtiques" de la Gaule ${ }^{32}$.

\section{Patrick Galliou et Michel Cúminat}

30 Certains de ces chenets ont été découverts pres de fours de potiers ou dans de grands centres de production de corramiques, cf. J. Díchlitetre, L.e bélier..., op. cil., p. $72, \mathrm{n}^{\circ} 10 ;$ p. $72-73, \mathrm{n}^{\circ} 12$; p. $73, n^{\circ} 13 ;$ p. $75, n^{\circ} 18$.

31 Cf. en parliculier : M. CLiswr, Lne imporialion précoce de céramique commune en Armorique: les urnes globulaires à hord mouluré, dans Archéologie en Bretayne, 23,1979, p. 13-17.

32 Sous remercions la birection des Antiquites historiques de Bretagne qui nous a permis d'utiliser le dessin des deux chenets, réalisé par ses soins. 